\title{
Tychonoff Objects in the Topological Category of Cauchy Spaces
}

\author{
Muammer Kula* and Sümeyye Kula
}

(Communicated by Ishak ALTUN)

\begin{abstract}
There are various forms of Tychonoff objects for an arbitrary set-based topological category. In this paper, any explicit characterization of each of the Tychonoff Objects is given in the topological category of Cauchy spaces. Moreover, we characterize each of them for the category of Cauchy spaces and investigate the relationships among the various $T_{i}, i=0,1,2,3,4, \operatorname{Pre} T_{2}$, and $\mathbf{T}_{2}$ (we will refer to it as the usual one) structures are examined in this category.
\end{abstract}

Keywords: Topological category; Cauchy space; Cauchy map; Separation.

AMS Subject Classification (2010): Primary: 54B30 ; Secondary: 54D10; 54A05; 54A20; $18 B 99$.

${ }^{*}$ Corresponding author

\section{Introduction}

In general topology and analysis, a Cauchy space is a generalization of metric spaces and uniform spaces. The theory of Cauchy spaces was initiated by H. J. Kowalsky [20]. Cauchy spaces were introduced by H. Keller [17] in 1968.

In 1970, the study of regular Cauchy completions was initiated by J. Ramaley and O. Wyler [31]. Later, D. C. Kent and G. D. Richardson $([18,19])$ characterized the $T_{3}$ Cauchy spaces which have $T_{3}$ completions and constructed a regular completion functor.

In 1968, Keller [17] introduced the axiomatic definition of Cauchy spaces, which is given briefly in the preliminaries section.

Filter spaces are generalizations of Cauchy spaces. If we exclude the last of three Keller's [17] axioms for a Cauchy space, then the resulting space is what we call a filter space. In [13], it is shown that the category FIL of filter spaces is isomorphic to the category of filter meretopic spaces which were introduced by Katětov [16]. The category of Cauchy spaces is also known to be a bireflective, finally dense subcategory of FIL [30].

All our preliminary information on Cauchy spaces and more information can be found in [24].

The notions of "closedness" and "strong closedness" in set based topological categories are introduced by Baran $[2,4]$ and it is shown in [9] that these notions form an appropriate closure operator in the sense of Dikranjan and Giuli [14] in some well-known topological categories. Moreover, various generalizations of each of $T_{i}, i=0,1,2$ separation properties for an arbitrary topological category over $\boldsymbol{S e t}$, the category of sets are given and the relationship among various forms of each of these notions are investigated by Baran in $[2,7,8,10,11]$.

The main goal of this paper is

1. to give the characterization of each of the Tychonoff objects in the topological category of Cauchy spaces,

2. to examine how these generalizations are related, and

Received : 02-05-2018, Accepted : 14-08-2018 
3. to show that specific relationships that arise among the various $T_{i}, i=0,1,2,3,4, \operatorname{PreT}_{2}$, and $\mathbf{T}_{2}$ (we will refer to it as the usual one) structures are examined in the topological category of Cauchy spaces.

\section{Preliminaries}

The followings are some basic definitions and notations which we will use throughout the paper.

Let $\mathcal{E}$ and $\mathcal{B}$ be any categories. The functor $\mathcal{U}: \mathcal{E} \longrightarrow \mathcal{B}$ is said to be topological or that $\mathcal{E}$ is a topological category over $\mathcal{B}$ if $\mathcal{U}$ is concrete (i.e., faithful, amnestic and transportable), has small (i.e., sets) fibers, and for which every $\mathcal{U}$-source has an initial lift or, equivalently, for which each $\mathcal{U}$-sink has a final lift [1].

Note that a topological functor $\mathcal{U}: \mathcal{E} \longrightarrow \mathcal{B}$ is said to be normalized if constant objects, i.e., subterminals, have a unique structure $[1,5,10,26,29]$.

Recall in [1,29], that an object $X \in \mathcal{E}$ (where $X \in \mathcal{E}$ stands for $X \in \mathrm{Ob} \mathcal{E}$ ), a topological category, is discrete iff every map $\mathcal{U}(X) \rightarrow \mathcal{U}(Y)$ lifts to a map $X \rightarrow Y$ for each object $Y \in \mathcal{E}$ and an object $X \in \mathcal{E}$ is indiscrete iff every map $\mathcal{U}(Y) \rightarrow \mathcal{U}(X)$ lifts to a map $Y \rightarrow X$ for each object $Y \in \mathcal{E}$.

Let $\mathcal{E}$ be a topological category and $X \in \mathcal{E}$. $A$ is called a subspace of $X$ if the inclusion map $i: A \rightarrow X$ is an initial lift (i.e., an embedding) and we denote it by $A \subset X$.

A filter on a set $X$ is a collection of subsets of $X$, containing $X$, which is closed under finite intersection and formation of supersets (it may contain $\emptyset$ ). Let $\mathbf{F}(X)$ denote the set of filters on $X$. If $\alpha, \beta \in \mathbf{F}(X)$, then $\beta \geq \alpha$ if and only if for each $\mathrm{U} \in \alpha, \exists \mathrm{V} \in \beta$ such that $V \subseteq U$, that is equivalent to $\beta \supset \alpha$. This defines a partial order relation on $\mathbf{F}(X) \cdot \dot{x}=[\{x\}]$ is the filter generated by the singleton set $\{x\}$ where $[\cdot]$ means generated filter and $\alpha \cap \beta=$ $[\{\mathrm{U} \cup \mathrm{V} \mid \mathrm{U} \in \alpha, \mathrm{V} \in \beta\}]$. If $\mathrm{U} \cap \mathrm{V} \neq \emptyset$, for all $\mathrm{U} \in \alpha$ and $\mathrm{V} \in \beta$, then $\alpha \vee \beta$ is the filter $[\{\mathrm{U} \cap \mathrm{V} \mid \mathrm{U} \in \alpha, \mathrm{V} \in \beta\}]$. If $\exists \mathrm{U} \in \alpha$ and $\mathrm{V} \in \beta$ such that $\mathrm{U} \cap \mathrm{V}=\emptyset$, then we say that $\alpha \vee \beta$ fails to exist.

Let $A$ be a set and $q$ be a function on $A$ that assigns to each point $x$ of $A$ a set of filters (proper or not, where a filter $\delta$ is proper iff $\delta$ does not contain the empty set, $\emptyset$, i.e., $\delta \neq[\emptyset]$ ) (the filters converging to $x$ ) is called a convergence structure on $A((A, q)$ a convergence space (in [29], it is called a convergence space)) iff it satisfies the following three conditions ([28] p. 1374 or [29] p. 142):

1. $[x]=[\{x\}] \in q(x)$ for each $x \in A$ (where $[F]=\{B \subset A: F \subset B\}$ ).

2. $\beta \supset \alpha \in q(x)$ implies $\beta \in q(x)$ for any filter $\beta$ on $A$.

3. $\alpha \in q(x) \Rightarrow \alpha \cap[x] \in q(x)$.

A map $f:(A, q) \rightarrow(B, s)$ between two convergence spaces is called continuous iff $\alpha \in q(x)$ implies $f(\alpha) \in$ $s(f(x))$ (where $f(\alpha)$ denotes the filter generated by $\{f(D): D \in \alpha\})$. The category of convergence spaces and continuous maps is denoted by Con (in [29] Conv).

For filters $\alpha$ and $\beta$ we denote by $\alpha \cup \beta$ the smallest filter containing both $\alpha$ and $\beta$.

Definition 2.1. (cf. [17]) Let $A$ be a set and $K \subset \mathbf{F}(A)$ be subject to the following axioms:

1. $[x]=[\{x\}] \in K$ for each $x \in A$ (where $[x]=\{B \subset A: x \in B\}$ );

2. $\alpha \in K$ and $\beta \geq \alpha$ implies $\beta \in K$ (i.e., $\beta \supset \alpha \in K$ implies $\beta \in K$ for any filter $\beta$ on $A$ );

3. if $\alpha, \beta \in K$ and $\alpha \vee \beta$ exists (i.e., $\alpha \cup \beta$ is proper), then $\alpha \cap \beta \in K$.

Then $K$ is a precauchy (Cauchy) structure if it obeys 1-2 (resp. 1-3) and the pair $(A, K)$ is called a precauchy space (Cauchy space), resp. Members of $K$ are called Cauchy filters. A map $f:(A, K) \rightarrow(B, L)$ between Cauchy spaces is said to be Cauchy continuous (Cauchy map) iff $\alpha \in K$ implies $f(\alpha) \in L$ (where $f(\alpha)$ denotes the filter generated by $\{f(D): D \in \alpha\}$ ). The concrete category whose objects are the precauchy (Cauchy) spaces and whose morphisms are the Cauchy continuous maps is denoted by PCHY (CHY), respectively.

Definition 2.2. A source $\left\{f_{i}:(A, K) \rightarrow\left(A_{i}, K_{i}\right), i \in I\right\}$ in CHY is an initial lift iff $\alpha \in K$ precisely when $f_{i}(\alpha) \in$ $K_{i}$ for all $i \in I[24,30,32]$.

Definition 2.3. An epimorphism $f:(A, K) \rightarrow(B, L)$ in CHY (equivalently, $f$ is surjective) is a final lift iff $\alpha \in L$ implies that there exists a finite sequence $\alpha_{1}, \ldots, \alpha_{n}$ of Cauchy filters in $K$ such that every member of $\alpha_{i}$ intersects every member of $\alpha_{i+1}$ for all $i<n$ and such that $\stackrel{n}{n=1}_{n}^{n} f\left(\alpha_{i}\right) \subset \alpha[24,30,32]$.

Definition 2.4. Let $B$ be set and $p \in B$. Let $B \vee_{p} B$ be the wedge at $p$ ([2] p. 334), i.e., two disjoint copies of $B$ identified at $p$, i.e., the pushout of $p: 1 \rightarrow B$ along itself (where 1 is the terminal object in Set). An epi sink $\left\{i_{1}, i_{2}:(B, K) \rightarrow\left(B \vee_{p} B, L\right)\right\}$, where $i_{1}, i_{2}$ are the canonical injections, in CHY is a final lift if and only if the following statement holds. For any filter $\alpha$ on the wedge $B \vee_{p} B$, where either $\alpha \supset i_{k}\left(\alpha_{1}\right)$ for some $k=1,2$ and some $\alpha_{1} \in K$, or $\alpha \in L$, we have that there exist Cauchy filters $\alpha_{1}, \alpha_{2} \in K$ such that every member of $\alpha_{1}$ intersects every member of $\alpha_{2}$ (i.e., $\alpha_{1} \cup \alpha_{2}$ is proper) and $\alpha \supset i_{1} \alpha_{1} \cap i_{2} \alpha_{2}$. This is a special case of Definition 2.3. 
Definition 2.5. The discrete structure $(A, K)$ on $A$ in CHY is given by $K=\{[a] \mid a \in A\} \cup\{[\emptyset]\}[24,30]$.

Definition 2.6. The indiscrete structure $(A, K)$ on $A$ in CHY is given by $K=F(A)[24,30]$.

CHY is a normalized topological category. The category of Cauchy spaces is cartesian closed, and contains the category of uniform spaces as a full subcategory [30].

\section{3. $T_{2}$-Objects}

Recall, in $[2,11]$, that there are various ways of generalizing the usual $T_{2}$ separation axiom to topological categories. Moreover, the relationships among various forms of $T_{2}$-objects are established in [11].

Let $B$ be a nonempty set, $B^{2}=B \times B$ be cartesian product of $B$ with itself and $B^{2} \vee_{\Delta} B^{2}$ be two distinct copies of $B^{2}$ identified along the diagonal. A point $(x, y)$ in $B^{2} \vee_{\Delta} B^{2}$ will be denoted by $(x, y)_{1}\left(\right.$ or $\left.(x, y)_{2}\right)$ if $(x, y)$ is in the first (or second) component of $B^{2} \vee_{\Delta} B^{2}$, respectively. Clearly, $(x, y)_{1}=(x, y)_{2}$ iff $x=y$ [2].

The principal axis map $A: B^{2} \vee_{\Delta} B^{2} \rightarrow B^{3}$ is given by $A(x, y)_{1}=(x, y, x)$ and $A(x, y)_{2}=(x, x, y)$. The skewed axis map $S: B^{2} \vee_{\Delta} B^{2} \rightarrow B^{3}$ is given by $S(x, y)_{1}=(x, y, y)$ and $S(x, y)_{2}=(x, x, y)$ and the fold map, $\nabla: B^{2} \vee_{\Delta} B^{2} \rightarrow B^{2}$ is given by $\nabla(x, y)_{i}=(x, y)$ for $i=1,2$. Note that $\pi_{1} S=\pi_{11}=\pi_{1} A, \pi_{2} S=\pi_{21}=\pi_{2} A$, $\pi_{3} A=\pi_{12}$, and $\pi_{3} S=\pi_{22}$, where $\pi_{k}: B^{3} \rightarrow B$ the $k$-th projection $k=1,2,3$ and $\pi_{i j}=\pi_{i}+\pi_{j}: B^{2} \vee_{\Delta} B^{2} \rightarrow B$, for $i, j \in\{1,2\}$ [2].

Definition 3.1. (cf. $[2,4,10,11])$ Let $\mathcal{U}: \mathcal{E} \rightarrow \boldsymbol{S e t}$ be a topological functor, $X$ an object in $\mathcal{E}$ with $\mathcal{U}(X)=B$.

1. $X$ is $\overline{T_{0}}$ iff the initial lift of the $\mathcal{U}$-source $\left\{A: B^{2} \vee_{\Delta} B^{2} \rightarrow \mathcal{U}\left(X^{3}\right)=B^{3}\right.$ and $\left.\nabla: B^{2} \vee_{\Delta} B^{2} \rightarrow \mathcal{U D}\left(B^{2}\right)=B^{2}\right\}$ is discrete, where $\mathcal{D}$ is the discrete functor which is a left adjoint to $\mathcal{U}$.

2. $X$ is $T_{0}^{\prime}$ iff the initial lift of the $\mathcal{U}$-source $\left\{i d: B^{2} \vee_{\Delta} B^{2} \rightarrow \mathcal{U}\left(B^{2} \vee_{\Delta} B^{2}\right)^{\prime}=B^{2} \vee_{\Delta} B^{2}\right.$ and $\nabla: B^{2} \vee_{\Delta} B^{2} \rightarrow$ $\left.\mathcal{U D}\left(B^{2}\right)=B^{2}\right\}$ is discrete, where $\left(B^{2} \vee_{\Delta} B^{2}\right)^{\prime}$ is the final lift of the $\mathcal{U}$-sink $\left\{i_{1}, i_{2}: \mathcal{U}\left(X^{2}\right)=B^{2} \rightarrow B^{2} \vee_{\Delta} B^{2}\right\}$ and $\mathcal{D}\left(B^{2}\right)$ is the discrete structure on $B^{2}$. Here, $i_{1}$ and $i_{2}$ are the canonical injections.

3. $X$ is $T_{0}$ iff $X$ does not contain an indiscrete subspace with (at least) two points [25,34].

4. $X$ is $T_{1}$ iff the initial lift of the $\mathcal{U}$-source $\left\{S: B^{2} \vee_{\Delta} B^{2} \rightarrow \mathcal{U}\left(X^{3}\right)=B^{3}\right.$ and $\left.\nabla: B^{2} \vee_{\Delta} B^{2} \rightarrow \mathcal{U D}\left(B^{2}\right)=B^{2}\right\}$ is discrete.

5. $X$ is $\operatorname{Pre} \overline{T_{2}}$ iff the initial lifts of the $\mathcal{U}$-source $\left\{A: B^{2} \vee_{\Delta} B^{2} \rightarrow \mathcal{U}\left(X^{3}\right)=B^{3}\right\}$ and $\left\{S: B^{2} \vee_{\Delta} B^{2} \rightarrow \mathcal{U}\left(X^{3}\right)=\right.$ $\left.B^{3}\right\}$ coincide.

6. $X$ is $\operatorname{PreT}_{2}^{\prime}$ iff the initial lift of the $\mathcal{U}$-source $\left\{S: B^{2} \vee_{\Delta} B^{2} \rightarrow \mathcal{U}\left(X^{3}\right)=B^{3}\right\}$ and the final lift of the $\mathcal{U}$-sink $\left\{i_{1}, i_{2}: \mathcal{U}\left(X^{2}\right)=B^{2} \rightarrow B^{2} \vee_{\Delta} B^{2}\right\}$ coincide, where $i_{1}$ and $i_{2}$ are the canonical injections.

7. $X$ is $\overline{T_{2}}$ iff $X$ is $\overline{T_{0}}$ and $\operatorname{Pre} \overline{T_{2}}$.

8. $X$ is $T_{2}^{\prime}$ iff $X$ is $T_{0}^{\prime}$ and $\operatorname{Pre} T_{2}^{\prime}$.

9. $X$ is $S T_{2}$ iff $\Delta$, the diagonal, is strongly closed in $X^{2}$.

10. $X$ is $\Delta T_{2}$ iff $\Delta$, the diagonal, is closed in $X^{2}$.

11. $X$ is $K T_{2}$ iff $X$ is $T_{0}^{\prime}$ and $\operatorname{Pre} \overline{T_{2}}$.

12. $X$ is $L T_{2}$ iff $X$ is $\overline{T_{0}}$ and $P r e T_{2}^{\prime}$.

13. $X$ is $M T_{2}$ iff $X$ is $T_{0}$ and $\operatorname{Pre} T_{2}^{\prime}$.

14. $X$ is $N T_{2}$ iff $X$ is $T_{0}$ and $\operatorname{Pre} \overline{T_{2}}$.

Remark 3.1. Note that for the category Top of topological spaces, $\overline{T_{0}}, T_{0}^{\prime}, T_{0}$, or $T_{1}$, or $\operatorname{Pr} e \overline{T_{2}}, \operatorname{Pre} T_{2}^{\prime}$, or all of the $T_{2}$ 's in Definition 3.1 reduce to the usual $T_{0}$, or $T_{1}$, or $\operatorname{Pre}_{2} T_{2}$ (where a topological space is called $\operatorname{Pre} T_{2}$ if for any two distinct points, if there is a neighbourhood of one missing the other, then the two points have disjoint neighbourhoods), or $T_{2}$ separation axioms, respectively [2].

Definition 3.2. A Cauchy space $(A, K)$ is said to be $\mathbf{T}_{2}$ (we will refer to it as the usual one) if and only if $x=y$, whenever $[x] \cap[y] \in K[33]$. 
Theorem 3.1. (cf. [21]) Let $(A, K)$ be a Cauchy space. Then,

(1) $(A, K)$ in $\mathbf{C H Y}$ is $\bar{T}_{0}$ iff it is $T_{0}$ iff it is $T_{1}$ iff for each distinct pair $x$ and $y$ in $A$, we have $[x] \cap[y] \notin K$.

(2) All objects $(A, K)$ in $\mathbf{C H Y}$ are $T_{0}^{\prime}$.

(3) All objects $(A, K)$ in $\mathbf{C H Y}$ are $\operatorname{Pre}_{2}$.

(4) $(A, K)$ is Pre $T_{2}^{\prime}$ iff for each pair of distinct points $x$ and $y$ in $A$, we have $[x] \cap[y] \in K$ (equivalently, for each finite subset $F$ of $A$, we have $[F] \in K)$.

(5) $(A, K)$ is $\bar{T}_{2}$ iff for each distinct pair $x$ and $y$ in $A$, we have $[x] \cap[y] \notin K$.

(6) $(A, K)$ is $T_{2}^{\prime}$ iff for each distinct points $x$ and $y$ in $A$, we have $[x] \cap[y] \in K$ (equivalently, for each finite subset $F$ of $A$, we have $[F] \in K)$.

Remark 3.2. (cf. [21])

(1) If a Cauchy space $(A, K)$ is $\bar{T}_{0}$ or $T_{0}\left(T_{1}\right)$ then it is $T_{0}^{\prime}$. However, the converse is not true generally. For example, let $A=\{x, y\}$ and $K=\{[x],[y],[\{x, y\}],[\emptyset]\}$. Then $(A, K)$ is $T_{0}^{\prime}$ but it is not $\bar{T}_{0}$ or $T_{0}\left(T_{1}\right)$.

(2) If a Cauchy space $(A, K)$ is $\operatorname{Pre}_{2}^{\prime}$ then it is $\operatorname{Pre}_{2}$. However, the converse is not true, in general. For example, let $A=\{x, y\}$ and $K=\{[x],[y],[\emptyset]\}$. Then $(A, K)$ is $\operatorname{Pre}_{2}$ but it is not $\operatorname{PreT}_{2}^{\prime}$.

Remark 3.3. $(A, K)$ be in CHY. By Theorem 3.1, the following are equivalent:

(a) $(A, K)$ is $\bar{T}_{2}$ and $T_{2}^{\prime}$.

(b) $A$ is a point or the empty set [21].

Corollary 3.1. Let $(A, K)$ be in $\mathbf{C H Y}$. $(A, K)$ is $S T_{2}$ iff it is $\Delta T_{2}$ iff for each pair of distinct points $x$ and $y$ in $A$ and for any $\alpha, \beta \in K, \alpha \cup \beta$ is improper if $\alpha \subset[x]$ and $\beta \subset[y][21]$.

Remark 3.4. ( $A, K)$ be in CHY. By Remark 4.5 (2) of [22], $(A, K)$ is $\bar{T}_{2}$ iff $(A, K)$ is $S T_{2}$ or $\Delta T_{2}$.

Remark 3.5. ([3], p. 106) Let $\alpha$ and $\beta$ be filters on $A$. If $f: A \rightarrow B$ is a function, then $f(\alpha \cap \beta)=f \alpha \cap f \beta$.

Let $(A, K)$ be in $\mathbf{C H Y}$, and $F$ be a nonempty subset of $A$. Let $q:(A, K) \rightarrow(A / F, L)$ be the quotient map that identifying $F$ to a point, * [2].

Theorem 3.2. (cf. [23])

(1) If $(A, K)$ is $T_{2}^{\prime}$, then $(A / F, L)$ is $T_{2}^{\prime}$.

(2) If $(A, K)$ is $\bar{T}_{2}$, then $(A / F, L)$ is $\bar{T}_{2}$.

(3) If $(A, K)$ is $\operatorname{Pre} \bar{T}_{2}$, then $(A / F, L)$ is $\operatorname{Pre}_{2}$.

(4) If $(A, K)$ is $\operatorname{Pre} T_{2}^{\prime}$, then $(A / F, L)$ is $\operatorname{PreT}_{2}^{\prime}$

Theorem 3.3. Let $(A, K)$ be in CHY. $\emptyset \neq F \subset A$ is closed iff for each $a \in A$ with $a \notin F$ and for all $\alpha \in K, \alpha \cup[F]$ is improper or $\alpha \nsubseteq[a][21]$.

Theorem 3.4. Let $(A, K)$ be in CHY. $\emptyset \neq F \subset A$ is strongly closed iff for each $a \in A$ with $a \notin F$ and for all $\alpha \in K$, $\alpha \cup[F]$ is improper or $\alpha \nsubseteq[a][21]$.

Theorem 3.5. (cf. [23])

(1) If $(A, K)$ is $S T_{2}$ (or $\left.\Delta T_{2}\right)$ and $F$ is (strongly) closed, then $(A / F, L)$ is $S T_{2}$ (or $\Delta T_{2}$ ).

(2) All objects $(A, K)$ in $\mathbf{C H Y}$ are $K T_{2}$.

(3) $(A, K)$ in $\mathbf{C H Y}$ is $L T_{2}$ iff $A$ is a point or the empty set.

(4) $(A, K)$ in $\mathbf{C H Y}$ is $M T_{2}$ iff $A$ is a point or the empty set.

(5) $(A, K)$ in $\mathbf{C H Y}$ is $N T_{2}$ iff for each distinct pair $x$ and $y$ in $A,[x] \cap[y] \notin K$.

Remark 3.6. (cf. [23])

(1) If a Cauchy space $(A, K)$ is $L T_{2}\left(M T_{2}\right)$ then it is $K T_{2}$. However, the converse is not true, in general. For example, let $A=\{x, y\}$ and $K=\left\{[x],[y]\right.$, [Ø] . Then $(A, K)$ is $K T_{2}$ but it is not $L T_{2}\left(M T_{2}\right)$.

(2) If a Cauchy space $(A, K)$ is $N T_{2}$ then it is $K T_{2}$. However, the converse is not true, in general. For example, let $A=\{x, y\}$ and $K=\{[x],[y],[\{x, y\}],[\emptyset]\}$. Then $(A, K)$ is $K T_{2}$ but it is not $N T_{2}$.

(3) If a Cauchy space $(A, K)$ is $L T_{2}\left(M T_{2}\right)$ then it is $N T_{2}$. However, the converse is not true, in general. For example, let $A=\{x, y\}$ and $K=\{[x],[y],[\emptyset]\}$. Then $(A, K)$ is $N T_{2}$ but it is not $L T_{2}\left(M T_{2}\right)$. 
Theorem 3.6. Let $(A, K)$ be a Cauchy space and $B \subset A$.

(1) If $(A, K)$ is $\operatorname{Pre}_{2}$, then $\left(B, K_{1}\right)$ is also $\operatorname{Pre}_{2}$.

(2) If $(A, K)$ is $\operatorname{Pre}_{2}^{\prime}$, then $\left(B, K_{1}\right)$ is also $\operatorname{Pre}_{2}^{\prime}$.

(3) If $(A, K)$ is $\bar{T}_{2}$, then $\left(B, K_{1}\right)$ is also $\bar{T}_{2}$.

(4) If $(A, K)$ is $T_{2}^{\prime}$, then $\left(B, K_{1}\right)$ is also $T_{2}^{\prime}$.

Proof. Let $f: B \hookrightarrow A$ be the inclusion map defined by $f(x)=x$ for $x \in B$ and $K_{1}$ be the initial lift of $f: B \hookrightarrow(A, K)$.

(1) Suppose that $(A, K)$ is $\operatorname{Pre} \bar{T}_{2}$ and $x \in B$. By Definition 2.2 and Theorem 3.1(3), $\left(B, K_{1}\right)$ is also $\operatorname{Pre} \bar{T}_{2}$.

(2) Let $(A, K)$ is $\operatorname{PreT}_{2}^{\prime}$ and $x, y$ be any two distinct points of $B$. Since $B \subset A$ and $(A, K)$ is $\operatorname{Pre} T_{2}^{\prime}$, by Theorem 3.1 (4), we have $[x] \cap[y] \in K$ and $f([x] \cap[y])=f([x]) \cap f([y])=[x] \cap[y] \in K$. Hence by Definition 2.2, $[x] \cap[y] \in K_{1}$ and by Theorem $3.1(4),\left(B, K_{1}\right)$ is $\operatorname{PreT}_{2}^{\prime}$.

(3) Suppose that $(A, K)$ is $\bar{T}_{2}$ and $x, y$ be any two distinct points of $B$. Since $B \subset A$ and $(A, K)$ is $\bar{T}_{2}$, by Theorem 3.1 (5), we have $[x] \cap[y] \notin K$ and $f([x] \cap[y])=f([x]) \cap f([y])=[x] \cap[y] \notin K$. Hence by Definition 2.2, $[x] \cap[y] \notin K_{1}$ and by Theorem $3.1(5),\left(B, K_{1}\right)$ is $\bar{T}_{2}$.

The proof (4) is similar to the proof of (2) by using Theorem 3.1 (6).

\section{4. $T_{3}$-Objects}

We now recall, $([2,7,12])$, various generalizations of the usual $T_{3}$ separation axiom to arbitrary set based topological categories and characterize each of them for the topological categories CHY.

Definition 4.1. (cf. $[2,7,12])$ Let $\mathcal{U}: \mathcal{E} \rightarrow \boldsymbol{S e t}$ be a topological functor, $X$ an object in $\mathcal{E}$ with $\mathcal{U}(X)=B$. Let $F$ be a non-empty subset of $B$.

1. $X$ is $S \overline{T_{3}}$ iff $X$ is $T_{1}$ and $X / F$ is $\operatorname{Pre} \overline{T_{2}}$ for all strongly closed $F \neq \emptyset$ in $U(X)$.

2. $X$ is $S T_{3}^{\prime}$ iff $X$ is $T_{1}$ and $X / F$ is $P r e T_{2}^{\prime}$ for all strongly closed $F \neq \emptyset$ in $U(X)$.

3. $X$ is $\overline{T_{3}}$ iff $X$ is $T_{1}$ and $X / F$ is $\operatorname{Pr} e \overline{T_{2}}$ for all closed $F \neq \emptyset$ in $U(X)$.

4. $X$ is $T_{3}^{\prime}$ iff $X$ is $T_{1}$ and $X / F$ is $\operatorname{Pre}_{2}^{\prime}$ for all closed $F \neq \emptyset$ in $U(X)$.

5. $X$ is $K T_{3}$ iff $X$ is $T_{1}$ and $X / F$ is $\operatorname{Pre} \overline{T_{2}}$ if it is $T_{1}$, where $F \neq \emptyset$ in $U(X)$.

6. $X$ is $L T_{3}$ iff $X$ is $T_{1}$ and $X / F$ is $\operatorname{Pre}_{2}^{\prime}$ if it is $T_{1}$, where $F \neq \emptyset$ in $U(X)$.

7. $X$ is $S T_{3}$ iff $X$ is $T_{1}$ and $X / F$ is $S T_{2}$ if it is $T_{1}$, where $F \neq \emptyset$ in $U(X)$.

8. $X$ is $\Delta T_{3}$ iff $X$ is $T_{1}$ and $X / F$ is $\Delta T_{2}$ if it is $T_{1}$, where $F \neq \emptyset$ in $U(X)$.

Remark 4.1. 1. For the category Top of topological spaces, all of the $T_{3}$ 's reduce to the usual $T_{3}$ separation axiom (cf. $[2,12 ?])$.

2. If $\mathcal{U}: \mathcal{E} \rightarrow \boldsymbol{B}$, where $\boldsymbol{B}$ is a topos [15], then Parts (1), (2), and (5)-(8)of Definition 4.1 still make sense since each of these notions requires only finite products and finite colimits in their definitions. Furthermore, if $\boldsymbol{B}$ has infinite products and infinite wedge products, then Definition 4.1 (4), also, makes sense.

Theorem 4.1. (cf. [23])

(1) $(A, K)$ in $\mathbf{C H Y}$ is $S \bar{T}_{3}$ iff for each distinct pair $x$ and $y$ in $A,[x] \cap[y] \notin K$.

(2) $(A, K)$ in $\mathbf{C H Y}$ is $S T_{3}^{\prime}$ iff $A$ is a point or the empty set.

(3) $(A, K)$ in $\mathbf{C H Y}$ is $\bar{T}_{3}$ iff for each distinct pair $x$ and $y$ in $A,[x] \cap[y] \notin K$.

(4) $(A, K)$ in $\mathbf{C H Y}$ is $T_{3}^{\prime}$ iff $A$ is a point or the empty set.

(5) $(A, K)$ in $\mathbf{C H Y}$ is $K T_{3}$ iff for each distinct pair $x$ and $y$ in $A,[x] \cap[y] \notin K$.

(6) $(A, K)$ in $\mathbf{C H Y}$ is $L T_{3}$ iff $A$ is a point or the empty set.

(7) $(A, K)$ in $\mathbf{C H Y}$ is $S T_{3}$ iff for each pair of distinct points $x$ and $y$ in $A$ and for any $\alpha, \beta \in K, \alpha \cup \beta$ is improper if $\alpha \subset[x]$ and $\beta \subset[y]$.

(8) $(A, K)$ in $\mathbf{C H Y}$ is $\Delta T_{3}$ iff for each pair of distinct points $x$ and $y$ in $A$ and for any $\alpha, \beta \in K, \alpha \cup \beta$ is improper if $\alpha \subset[x]$ and $\beta \subset[y]$. 
Theorem 4.2. If $(A, K)$ is $K T_{3}$, then $(A / F, L)$ is $K T_{3}$.

Proof. Suppose $(A, K)$ is $K T_{3}$. Let $a$ and $b$ be any distinct pair of points in $A / F$. By Theorem 4.1 (5), we only need to show that $[a] \cap[b] \notin L$, where $L$ is the structure on $A / F$ induced by $q$. Suppose that $a \neq *$ and $[a],[*] \in L$ implies $\exists[a],[y] \in K$ such that $[a] \supseteq q([a]),[*] \supseteq q([y])$, and $x=q x=a, q y=*$ for any $y \in F$. If $[a] \cap[*] \in L$, then $[a] \cap[y] \in K$, by definition of the quotient map and Remark 3.5. But $[a] \cap[y] \notin K$ since $(A, K)$ is $K T_{3}$. Hence $[a] \cap[*] \notin L$. Similarly, if $a \neq b \neq *$ and $[a],[b] \in L$ implies $\exists[a],[b] \in K$ such that $[a] \supseteq q([a]),[b] \supseteq q([b])$, and $x=q x=a, q b=b$. If $[a] \cap[b] \in L$, then $[a] \cap[b] \in K$, by definition of the quotient map and Remark 3.5. But $[a] \cap[b] \notin K$ since $(A, K)$ is $K T_{3}$. Hence $[a] \cap[b] \notin L$.

Consequently for each distinct points $a$ and $b$ in $A / F$, we have $[a] \cap[b] \notin L$. Hence by Theorem $4.1(5),(A / F, L)$ is $K T_{3}$.

Theorem 4.3. If $(A, K)$ is $\Delta T_{3}$, then $(A / F, L)$ is $\Delta T_{3}$.

Proof. It follows from Theorem 4.2.

\section{5. $T_{4}$-Objects}

We now recall various generalizations of the usual $T_{4}$ separation axiom to arbitrary set based topological categories that are defined in $[2,7,12]$, and characterize each of them for the topological categories CHY.

Definition 5.1. (cf. $[2,7,12]$ ) Let $\mathcal{U}: \mathcal{E} \rightarrow \boldsymbol{S e t}$ be a topological functor and $X$ an object in $\mathcal{E}$ with $\mathcal{U}(X)=B$. Let $F$ be a non-empty subset of $B$.

1. $X$ is $S \overline{T_{4}}$ iff $X$ is $T_{1}$ and $X / F$ is $S \overline{T_{3}}$ for all strongly closed $F \neq \emptyset$ in $U(X)$.

2. $X$ is $S T_{4}^{\prime}$ iff $X$ is $T_{1}$ and $X / F$ is $S T_{3}^{\prime}$ for all strongly closed $F \neq \emptyset$ in $U(X)$.

3. $X$ is $\overline{T_{4}}$ iff $X$ is $T_{1}$ and $X / F$ is $\overline{T_{3}}$ for all closed $F \neq \emptyset$ in $U(X)$.

4. $X$ is $T_{4}^{\prime}$ iff $X$ is $T_{1}$ and $X / F$ is $T_{3}^{\prime}$ for all closed $F \neq \emptyset$ in $U(X)$.

5. $X$ is $\Delta T_{4}$ iff $X$ is $T_{1}$ and $X / F$ is $\Delta T_{3}$ if it is $T_{1}$, where $F \neq \emptyset$ in $U(X)$.

6. $X$ is $K T_{4}$ iff $X$ is $T_{1}$ and $X / F$ is $K T_{3}$ if it is $T_{1}$, where $F \neq \emptyset$ in $U(X)$.

7. $X$ is $L T_{4}$ iff $X$ is $T_{1}$ and $X / F$ is $L T_{2}$ if it is $T_{1}$, where $F \neq \emptyset$ in $U(X)$.

Remark 5.1. 1. For the category Top of topological spaces, all of the $T_{4}$ 's reduce to the usual $T_{4}$ separation axiom $([2,7,12])$.

2. If $\mathcal{U}: \mathcal{E} \rightarrow \boldsymbol{B}$, where $\boldsymbol{B}$ is a topos [15], then Definition 5.1 still makes sense since each of these notions requires only finite products and finite colimits in their definitions.

Theorem 5.1. (cf. [23])

(1) $(A, K)$ in $\mathbf{C H Y}$ is $\overline{S T}_{4}$ iff for each distinct pair $x$ and $y$ in $A,[x] \cap[y] \notin K$.

(2) $(A, K)$ in $\mathbf{C H Y}$ is $S T_{4}^{\prime}$ iff $A$ is a point or the empty set.

(3) $(A, K)$ in $\mathbf{C H Y}$ is $\bar{T}_{4}$ iff for each distinct pair $x$ and $y$ in $A,[x] \cap[y] \notin K$.

(4) $(A, K)$ in $\mathbf{C H Y}$ is $T_{4}^{\prime}$ iff $A$ is a point or the empty set.

Theorem 5.2. $(A, K)$ in $\mathbf{C H Y}$ is $\Delta T_{4}$ iff for each pair of distinct points $x$ and $y$ in $A$ and for any $\alpha, \beta \in K, \alpha \cup \beta$ is improper if $\alpha \subset[x]$ and $\beta \subset[y]$.

Proof. It follows from Definition 5.1 (5), Theorem 3.1 (1) and Theorem 4.3.

Theorem 5.3. $(A, K)$ in $\mathbf{C H Y}$ is $K T_{4}$ iff for each distinct pair $x$ and $y$ in $A,[x] \cap[y] \notin K$.

Proof. It follows from Definition 5.1 (6), Theorem 3.1 (1) and Theorem 4.2.

Theorem 5.4. $(A, K)$ in $\mathbf{C H Y}$ is $L T_{4}$ iff $A$ is a point or the empty set.

Proof. It follows from Definition 5.1 (7) and Theorem 3.5 (3). 
Remark 5.2. Let $(A, K)$ be a Cauchy space. It follows from Theorem 3.5, Theorem 4.1, Theorem 5.1, Theorem 5.2 and Theorem 5.3 that $(A, K)$ is $N T 2$ iff $(A, K)$ is $S \bar{T}_{3}$ iff $(A, K)$ is $\bar{T}_{3}$ iff $(A, K)$ is $K T_{3}$ iff $(A, K)$ is $\overline{S T}_{4}$ iff $(A, K)$ is $\bar{T}_{4}$ iff $(A, K)$ is $K T_{4}$ iff $(A, K)$ is $\Delta T_{4}$ iff for each distinct pair $x$ and $y$ in $A,[x] \cap[y] \notin K$.

Remark 5.3. Let $(A, K)$ be a Cauchy space. It follows from Theorem 3.5, Theorem 4.1, Theorem 5.1 and Theorem 5.4 that $(A, K)$ is $S T_{3}^{\prime}$ iff $(A, K)$ is $T_{3}^{\prime}$ iff $(A, K)$ is $L T_{2}$ iff $(A, K)$ is $M T_{2}$ iff $(A, K)$ is $L T_{3}$ iff $(A, K)$ is $S T_{4}^{\prime}$ iff $(A, K)$ is $T_{4}^{\prime}$ iff $(A, K)$ is $L T_{4}$ iff $A$ is a point or the empty set.

\section{Tychonoff objects}

In this section, the characterization of Tychonoff objects in this category is given. Furthermore, we investigate the relationships between Tychonoff objects and $S T_{2}, \Delta T_{2}, S T_{3}, \Delta T_{3}$, generalized separation properties and separation properties at a point $\mathrm{p}$ in this category.

Definition 6.1. (cf. [7,12]) Let $\mathcal{U}: \mathcal{E} \longrightarrow S$ et be a topological functor and $X$ an object in $\mathcal{E}$ with $\mathcal{U}(X)=B$.

1. $X$ is $\Delta T_{3 \frac{1}{2}}$ iff $X$ is a subspace of $\Delta T_{4}$.

2. $X$ is $S T_{3 \frac{1}{2}}$ iff $X$ is a subspace of $S T_{4}$.

3. $X$ is $T_{3 \frac{1}{2}}^{\prime}$ iff $X$ is a subspace of $T_{4}^{\prime}$.

4. $X$ is $S T_{3 \frac{1}{2}}^{\prime}$ iff $X$ is a subspace of $S T_{4}^{\prime}$.

5. $X$ is $C \Delta T_{3 \frac{1}{2}}$ iff $X$ is a subspace of a compact $\Delta T_{2}$.

6. $X$ is $C S T_{3 \frac{1}{2}}$ iff $X$ is a subspace of a compact $S T_{2}$.

7. $X$ is $L T_{3 \frac{1}{2}}$ iff $X$ is a subspace of a compact $T_{2}^{\prime}$.

8. $X$ is $S \Delta T_{3 \frac{1}{2}}$ iff $X$ is a subspace of a strongly compact $\Delta T_{2}$.

9. $X$ is $S S T_{3 \frac{1}{2}}$ iff $X$ is a subspace of a strongly compact $S T_{2}$.

10. $X$ is $S L T_{3 \frac{1}{2}}$ iff $X$ is a subspace of a strongly compact $T_{2}^{\prime}$.

Remark 6.1. For the category Top of topological spaces, all six of the properties defined in Definition 6.1 are equivalent and reduce to the usual $T_{3 \frac{1}{2}}=$ Tychonoff, i.e, completely regular $T_{1}$ spaces [27], Remark 5.2, and Remark 6.2 .

Lemma 6.1. (cf. [21]) All objects in CHY are (strongly) compact.

Theorem 6.1. Let $(A, K)$ be a Cauchy space. Then the followings are equivalent:

(1) $(A, K)$ is $\Delta T_{3 \frac{1}{2}}$

(2) $(A, K)$ is $S T_{3 \frac{1}{2}}$,

(3) $(A, K)$ is $C \Delta T_{3 \frac{1}{2}}$,

(4) $(A, K)$ is $C S \Delta T_{3 \frac{1}{2}}$,

(5) $(A, K)$ is $S \Delta T_{3 \frac{1}{2}}$

(6) $(A, K)$ is $S S T_{3 \frac{1}{2}}$,

(7) for each distinct pair $x$ and $y$ in $A$, we have $[x] \cap[y] \notin K$.

Proof. It follows from Corollary 3.1, Theorem 5.1, Theorem 5.2, Definition 6.1 and Lemma 6.1.

Example 6.1. Let $X=\{a, b\}, \delta=\{(X, X),(\{a\},\{a\}),(\{b\},\{b\}),(X,\{a\}),(\{a\}, X),(X,\{b\}),(\{b\}, X)\}$ and $\delta_{1}=$ $\{(X, X),(\{a\},\{a\}),(\{b\},\{b\}),(X,\{a\}),(\{a\}, X),(X,\{b\}),(\{b\}, X),(\{a\},\{b\}),(\{b\},\{a\})\}$. Then $(X, \delta)$ is $C \Delta T_{3 \frac{1}{2}}$, but $\left(X, \delta_{1}\right)$ is not $C \Delta T_{3 \frac{1}{2}}$, since $(\{a\},\{b\}) \in \delta$ with $a \neq b$. 
Theorem 6.2. Let $(A, K)$ be a Cauchy space. Then the followings are equivalent:

(1) $(A, K)$ is $T_{3 \frac{1}{2}}^{\prime}{ }^{\prime}$

(2) $(A, K)$ is $S T_{3 \frac{1}{2}}^{\prime}$

(3) $A$ is a point or the empty set.

Proof. It follows from Theorem 5.1 and Definition 6.1.

Theorem 6.3. Let $(A, K)$ be a Cauchy space. Then the followings are equivalent:

(1) $(A, K)$ is $L T_{3 \frac{1}{2}}$,

(2) $(A, K)$ is $S L T_{3 \frac{1}{2}}$

(3) for each pair of distinct points $x$ and $y$ in $A$, we have $[x] \cap[y] \in K$ (equivalently, for each finite subset $F$ of $A$, we have $[F] \in K)$.

Proof. It follows from Theorem 3.1, Definition 6.1 and Lemma 6.1.

We can infer the following results.

Remark 6.2. Let $(A, K)$ be in CHY. The followings are equivalent;

1. By Theorems 3.1, 4.1 and 6.1, Corollary 3.1, $(A, K)$ is $T_{1}$ iff it is $T_{0}$ iff it is $\bar{T}_{0}$ iff $(A, K)$ is $S T_{3}$ iff it is $\bar{T}_{3}$ iff it is $K T_{3}$ iff $(A, K)$ is $\overline{S T}_{4}$ iff it is $\bar{T}_{4}$ iff $(A, K)$ is $S T_{2}$ or $\Delta T_{2}$ iff $(A, K)$ is $S T_{3}$ or $\Delta T_{3}$ iff $(A, K)$ is $N T 2$ iff $(A, K)$ is $\Delta T_{3 \frac{1}{2}}$ iff $(A, K)$ is $S T_{3 \frac{1}{2}}$ iff $(A, K)$ is $C \Delta T_{3 \frac{1}{2}}$ iff $(A, K)$ is $C S \Delta T_{3 \frac{1}{2}}$ iff $(A, K)$ is $S \Delta T_{3 \frac{1}{2}}$ iff $(A, K)$ is $S S T_{3 \frac{1}{2}}$.

2. By Theorems 3.1, 4.1 and 6.1, Corollary 3.1, $(A, K)$ is $\bar{T}_{2}$ iff $(A, K)$ is $\overline{S T}_{3}$ iff $(A, K)$ is $\bar{T}_{3}$ iff $(A, K)$ is $K T_{3}$ iff $(A, K)$ is $S \bar{T}_{4}$ iff $(A, K)$ is $\bar{T}_{4}$ iff $(A, K)$ is $S T_{2}$ or $\Delta T_{2}$ iff $(A, K)$ is $S T_{3}$ or $\Delta T_{3}$ iff $(A, K)$ is $N T 2$ iff $(A, K)$ is $\Delta T_{3 \frac{1}{2}}$ iff $(A, K)$ is $S T_{3 \frac{1}{2}}$ iff $(A, K)$ is $C \Delta T_{3 \frac{1}{2}}$ iff $(A, K)$ is $C S \Delta T_{3 \frac{1}{2}}$ iff $(A, K)$ is $S \Delta T_{3 \frac{1}{2}}$ iff $(A, K)$ is $S S T_{3 \frac{1}{2}}$.

3. By Theorems 3.1, 4.1 and 6.1, Corollary 3.1, if $(A, K)$ is $S \bar{T}_{3}$ or $\bar{T}_{3}$ or $K T_{3}$ or $S \bar{T}_{4}$ or $\bar{T}_{4}$ or $S T_{2}$ or $\Delta T_{2}$ or $S T_{3}$ or $\Delta T_{3}$ or $N T 2$ or $\Delta T_{3 \frac{1}{2}}$ or $S T_{3 \frac{1}{2}}$ or $C \Delta T_{3 \frac{1}{2}}$ or $C S \Delta T_{3 \frac{1}{2}}$ or $S \Delta T_{3 \frac{1}{2}}$ or $S S T_{3 \frac{1}{2}}$, then $(A, K)$ is $T_{0}^{\prime}$. But the converse of implication is not true, in general. For example, let $A=\{x, y\}$ and $K=\{[x],[y],[\{x, y\}],[\emptyset]\}$. Then $(A, K)$ is $T_{0}^{\prime}$ but it is not $\overline{S T}_{3}$ or $\bar{T}_{3}$ or $K T_{3}$ or $S \bar{T}_{4}$ or $\bar{T}_{4}$ or $S T_{2}$ or $\Delta T_{2}$ or $S T_{3}$ or $\Delta T_{3}$ or $N T 2$ or $\Delta T_{3 \frac{1}{2}}$ or $S T_{3 \frac{1}{2}}$ or $C \Delta T_{3 \frac{1}{2}}$ or $C S \Delta T_{3 \frac{1}{2}}$ or $S \Delta T_{3 \frac{1}{2}}$ or $S S T_{3 \frac{1}{2}}$.

4. By Theorems 3.1, 4.1 and 6.1, Corollary 3.1, if $(A, K)$ is $\overline{S T}_{3}$ or $\bar{T}_{3}$ or $K T_{3}$ or $S \bar{T}_{4}$ or $\bar{T}_{4}$ or $S T_{2}$ or $\Delta T_{2}$ or $S T_{3}$ or $\Delta T_{3}$ or $N T 2$ or $\Delta T_{3 \frac{1}{2}}$ or $S T_{3 \frac{1}{2}}$ or $C \Delta T_{3 \frac{1}{2}}$ or $C S \Delta T_{3 \frac{1}{2}}$ or $S \Delta T_{3 \frac{1}{2}}$ or $S S T_{3 \frac{1}{2}}$, then $(A, K)$ is $\operatorname{Pre} \bar{T}_{2}$. But the converse of implication is not true, in general. For example, let $A=\{x, y\}$ and $K=\{[x],[y],[\{x, y\}],[\emptyset]\}$. Then $(A, K)$ is $\operatorname{Pre}_{2}$ but it is not $\overline{S T}_{3}$ or $\bar{T}_{3}$ or $K T_{3}$ or $S \bar{T}_{4}$ or $\bar{T}_{4}$ or $S T_{2}$ or $\Delta T_{2}$ or $S T_{3}$ or $\Delta T_{3}$ or $N T 2$ or $\Delta T_{3 \frac{1}{2}}$ or $S T_{3 \frac{1}{2}}$ or $C \Delta T_{3 \frac{1}{2}}$ or $C S \Delta T_{3 \frac{1}{2}}$ or $S \Delta T_{3 \frac{1}{2}}$ or $S S T_{3 \frac{1}{2}}$.

5. By Theorems 3.1, 4.1 and 6.2, Corollary 3.1, the followings are equivalent:

(a) $(A, K)$ is $\operatorname{PreT}_{2}^{\prime}\left(T_{2}^{\prime}\right), L T_{3 \frac{1}{2}}, S L T_{3 \frac{1}{2}}$, and is $\overline{S T}_{3}$ or $\bar{T}_{3}$ or $K T_{3}$ or $S \bar{T}_{4}$ or $\bar{T}_{4}$ or $S T_{2}$ or $\Delta T_{2}$ or $S T_{3}$ or $\Delta T_{3}$ or $N T 2$ or $\Delta T_{3 \frac{1}{2}}$ or $S T_{3 \frac{1}{2}}$ or $C \Delta T_{3 \frac{1}{2}}$ or $C S \Delta T_{3 \frac{1}{2}}$ or $S \Delta T_{3 \frac{1}{2}}$ or $S S T_{3 \frac{1}{2}}$.

(b) $A$ is a point or the empty set.

6. By Theorems 3.1, 4.1 and 6.1, Corollary 3.1 and Definition 3.2, $(A, K)$ is $S \bar{T}_{3}$ or $\bar{T}_{3}$ or $K T_{3}$ or $S \bar{T}_{4}$ or $\bar{T}_{4}$ or $S T_{2}$ or $\Delta T_{2}$ or $S T_{3}$ or $\Delta T_{3}$ or $N T 2$ or $\Delta T_{3 \frac{1}{2}}$ or $S T_{3 \frac{1}{2}}$ or $C \Delta T_{3 \frac{1}{2}}$ or $C S \Delta T_{3 \frac{1}{2}}$ or $S \Delta T_{3 \frac{1}{2}}$ or $S S T_{3 \frac{1}{2}}$ iff $(A, K)$ is $\mathbf{T}_{2}$ (we will refer to it as the usual one).

\section{References}

[1] Adámek, J., Herrlich, H. and Strecker, G. E., Abstract and Concrete Categories, Wiley, New York, 1990.

[2] Baran, M., Separation properties. Indian J. Pure Appl. Math. 23 (1992), 333-341.

[3] Baran, M., Stacks and filters. Turkish J. of Math.-Doğa. 16 (1992), 95-108.

[4] Baran, M., The notion of closedness in topological categories. Comment. Math. Univ. Carolinae. 34 (1993), 383-395. 
[5] Baran, M., Generalized Local Separation Properties. Indian J. Pure Appl. Math. 25 (1994), 615-620.

[6] Baran, M., A notion of compactness in topological categories. Publ. Math. Debrecen. 50 (3-4), 221-234, 1997.

[7] Baran, M., $T_{3}$ and $T_{4}$-objects in topological categories. Indian J. Pure Appl. Math. 29 (1), 59-69, 1998.

[8] Baran, M., Closure operators in convergence spaces. Acta Math. Hungar. 87 (2000), 33-45.

[9] Baran, M., Compactness, perfectness, separation, minimality and closedness with respect to closure operators. Applied Categorical Structures. 10 (2002), 403-415.

[10] Baran, M., PreT ${ }_{2}$-objects in topological categories. Applied Categorical Structures. 17 (2009), 591-602, DOI 10.1007/s10485-008-9161-4.

[11] Baran, M. and Altındiş, H., T2-objects in topological categories. Acta Math. Hungar. 71 (1996), no. 1-2, 41-48.

[12] Baran, M. and Kula, M., A note on separation and compactness in categories of convergence spaces. Proceedings of the International Conference on Applicable General Topology (Ankara, 2001). Appl. Gen. Topol. 4 (1), 1-13, 2003.

[13] Bentley, H. L., Herrlich, H. and Lowen-Colebunders, E., Convergence. J. Pure Appl. Algebra. 68 (1990), no 1-2, 27-45.

[14] Dikranjan, D. and Giuli, E. Closure operators I. Topology Appl. 27 (1987), 129-143.

[15] Johnstone, P.T., Topos Theory. London Math. Soc. Monographs. No. 10, Academic Press, New York, 1977.

[16] Katětov, M., On continuity structures and spaces of mappings. Comm. Math. Univ. Car. 6 (1965), 257-278.

[17] Keller, H., Die Limes-uniformisierbarkeit der Limesräume. Math. Ann. 176 (1968), 334-341.

[18] Kent, D. C. and Richardson, G. D., Cauchy Spaces with Regular Completions. Pacific J. Math. 3 (1984), 105-116.

[19] Kent, D. C. and Richardson, G. D., Cauchy Completion Categories. Canad. Math. Bull. 32 (1989), 78-83.

[20] Kowalsky, H. J., Limesräume und Komplettierung. Math. Nachr. 12 (1954), 301-340.

[21] Kula, M., A Note on Cauchy Spaces. Acta Math. Hungar. 133 (2011), no. 1-2, 14-32, DOI:10.1007/s10474-0110136-9.

[22] Kula, M., Separation properties at $p$ for the topological category of Cauchy Spaces. Acta Math. Hungar. 136 (2012), no. 1-2, 1-15, DOI: 10.1007/s10474-012-0238-z.

[23] Kula, M., T3 and T4-Objects in the Topological Category of Cauchy Spaces. Communications, Series A1:Mathematics and Statistics. 66 (2017), no. 1, 29-42.

[24] Lowen-Colebunders, E., Function classes of Cauchy Continuous maps M. Dekker, New York, 1989.

[25] Marny, Th., Rechts-Bikategoriestrukturen in topologischen Kategorien. Dissertation, Freie Universität Berlin, 1973.

[26] Mielke, M.V., Separation axioms and geometric realizations. Indian J. Pure Appl. Math. 25 (1994), 711-722.

[27] Munkres, J. R., Topology:A First Course, Prentice-Hall, 1975.

[28] Nel, L. D., Initially structured categories and cartesian closedness. Canad. Journal of Math. XXVII (1975), 1361-1377.

[29] Preuss, G., Theory of Topological Structures. An Approach to Topological Categories., D. Reidel Publ. Co., Dordrecht, 1988.

[30] Preuss, G., Improvement of Cauchy spaces. QEA in General Topology. 9 (1991), 159-166.

[31] Ramaley, J. F. and Wyler, O., Cauchy Spaces II: Regular Completions and Compactifications, Math. Ann. 187 (1970), 187-199. 
[32] Rath, N., Precauchy spaces, PH.D. Thesis, Washington State University, 1994.

[33] Rath, N., Completion of a Cauchy space without the $T_{2}$-restriction on the space. Int. J. Math. Math. Sci. 3, 24 (2000), 163-172.

[34] Weck-Schwarz, S., $\mathrm{T}_{0}$-objects and separated objects in topological categories. Quaestiones Math. 14 (1991), 315-325.

\section{Affiliations}

MuAmmer Kula

AdDRESS: Erciyes University, Dept. of Mathematics, 38039, Kayseri-Turkey.

E-MAIL: kulam@erciyes.edu.tr

ORCID ID: 0000-0002-1366-6149

SÜMEYYE KULA

ADDRESS: Erciyes University, Dept. of Mathematics, 38039, Kayseri-Turkey..

E-MAIL: kulasbc@hotmail.com

ORCID ID: 0000-0001-5185-2877 